\title{
Organic Oxoacids
}

National Cancer Institute

\section{Source}

National Cancer Institute. Organic Oxoacids. NCI Thesaurus. Code C1947.

Organic acids that have oxygen atoms in the acidic groups. (NCI) 